\title{
The regulation of proteins associated with the cytoskeleton by hepatitis B virus X protein during hepatocarcinogenesis (Review)
}

\author{
FANYUN KONG ${ }^{*}$, HONGJUAN YOU* ${ }^{*}$ RENXIAN TANG and KUIYANG ZHENG \\ Jiangsu Key Laboratory of Immunity and Metabolism, Department of Pathogenic Biology and \\ Immunology, Xuzhou Medical University, Xuzhou, Jiangsu 221004, P.R. China
}

Received July 16, 2015; Accepted December 6, 2016

DOI: $10.3892 / \mathrm{ol} .2017 .5757$

\begin{abstract}
Hepatocellular carcinoma (HCC) is a major malignant disease worldwide, and chronic hepatitis $\mathrm{B}$ virus (HBV) infection is one of the primary causes for this type of cancer. Hepatitis B virus $\mathrm{X}$ protein $(\mathrm{HBx})$ is a non-structural protein encoded by the viral genome that has significant effects on the pathogenesis of HCC. With the development of high-throughput assays and technologies, the abnormal $\mathrm{HBx}$-induced expression of certain cellular proteins with assorted biological functions has been investigated. These target proteins identified by various methods include specific proteins associated with the cellular cytoskeleton, which contribute to $\mathrm{HBx}$-induced hepatocarcinogenesis. In addition, the cytoskeletal proteins deregulated by $\mathrm{HBx}$ are involved in cell morphogenesis, adhesion, migration and proliferation. This review aims to summarize the current understanding of the expression profiles of $\mathrm{HBx}$-associated cytoskeletal proteins, as well as their complex functions and underlying mechanisms in hepatocarcinogenesis. Considering that the potential therapeutics for various types of tumors may function through the stabilization of cytoskeletal proteins in order to restrict cellular movement and limit intracellular processes, clarifying the mechanisms underlying protein-associated cytoskeleton dysregulation by HBx may provide novel possibilities and potent therapeutic targets for HBV-associated HCC.
\end{abstract}

Correspondence to: Mrs. Renxian Tang, Jiangsu Key Laboratory of Immunity and Metabolism, Department of Pathogenic Biology and Immunology, Xuzhou Medical University, 209 Tongshan Road, Xuzhou, Jiangsu 221004, P.R. China

E-mail: tangrenxian-t@163.com

*Contributed equally

Key words: hepatitis $\mathrm{B}$ virus, hepatitis $\mathrm{B}$ virus $\mathrm{X}$ protein, hepatocellular carcinoma, cytoskeletal proteins

\section{Contents}

1. Introduction

2. The proteins associated with the cytoskeleton that are deregulated by HBx contribute to cellular morphological changes

3. The cellular cytoskeletal proteins associated with cell adhesion

4. The cellular cytoskeletal proteins associated with cellular migration

5. The cellular cytoskeletal proteins associated with cellular proliferation

6. Conclusion

\section{Introduction}

Hepatocellular carcinoma (HCC) is a major malignant disease globally, and patients with chronic hepatitis B virus (HBV) infection are at high risk of HCC development (1). The HBV genome contains four overlapping open reading frames (ORFs) that encode the viral surface proteins, core protein, viral polymerase and $\mathrm{X}$ protein ( $\mathrm{HBx}$ ) (2). Among these viral proteins, the surface proteins form the viral envelope, the core protein comprises the nucleocapsid and the viral polymerase is necessary for replication of the virus $(1,2)$. HBx, encoded by the smallest ORF, is a non-structural viral protein consisting of 154 amino acids with a molecular weight of $17 \mathrm{kDa}(1)$. Previous studies have indicated that HBx is a regulatory protein that benefits viral replication via interaction with numerous cellular proteins $(2,3)$. Furthermore, HBx has an important role in HBV-mediated $\mathrm{HCC}$ progression by interacting with transcription factors, activating cellular signaling pathways or inducing aberrant epigenetic changes in order to alter cellular gene expression patterns and to contribute to hepatocarcinogenesis through various underlying mechanisms (4-6). In addition, HBx mutants, in particular those with a $\mathrm{COOH}$-terminal deletion have been implicated in $\mathrm{HCC}$ and observed to participate in hepatocarcinogenesis (7).

With the development of high-throughput assays and technologies, including DNA array assays $(8,9)$, oligonucleotide microarray assays $(10,11)$, cDNA microarray technology $(12,13)$, ChIP-chip and expression microarray profiling (14-16), the abnormal changes in expression of 
various cellular proteins with biological activity induced by HBV (in particular by HBx) have been investigated. Of these target genes or proteins, those associated with the cellular cytoskeleton have been demonstrated to contribute to $\mathrm{HBx}$ induced hepatocarcinogenesis (9-15). The cytoskeleton is a structure that comprises several intracellular proteins that maintain cell shape, supply structural support and control cellular movement $(17,18)$. The cytoskeleton has three principal components: Microfilaments (or actin filaments), intermediate filaments and microtubules (17). The proteins associated with these cytoskeletal components participate in a number of cellular processes, including cell morphogenesis, adhesion, migration and proliferation $(17,18)$. Therefore, an improved understanding of the role and underlying mechanisms of cytoskeletal genes and proteins in $\mathrm{HBx}$-associated hepatocarcinogenesis may facilitate the identification of potential targets for the control and treatment of HBV-induced HCC. This review aims to summarize current knowledge of the cytoskeletal proteins that are deregulated by $\mathrm{HBx}$, in addition to their complex functions and associated underlying mechanisms in hepatocarcinogenesis.

\section{The proteins associated with the cytoskeleton that are deregulated by HBx contribute to cellular morphological changes}

Morphogenesis of mammalian cells involves marked changes in cell shape, mediated by rearrangements of cytoskeletal components (18). One important feature of the cytopathic effects (CPEs) mediated by viral infection is morphological changes in the host cells. Previous studies have demonstrated that HBV infection is able to induce CPEs in hepatocytes, with a ground glass appearance in the human liver/promoter-driven urokinase-type plasminogen activator/severe combined immunodeficiency mouse model or in cultured HeLa cells (19,20). Further studies reported that HBV-replicating HepG2 cells exhibited morphological changes and had the appearance of membrane rufflings and lamellipodia-like structures (21). The proteins belonging to the Rho GTPase family function as molecular switches and cycle from a GDP-bound inactive form to a GTP-bound active form (22). These proteins include ATP-dependent RNA helicase A, Ras-related C3 botulinum (Rac) and cell division control protein $42(\mathrm{Cdc} 42)$, which are implicated in regulating the assembly and organization of the cytoskeleton and are involved in cell morphogenesis $(21,22)$. Through implementing a cell-based HBV replication system, the shape change of cells infected with this virus has been proposed to be associated with the constitutively activated Rac substrate 1 (Rac1) (21). Notably, the interaction of Rac1 nucleotide exchange factor ( $\beta$ PIX) with HBx via an SRC homology 3 (SH3) domain-binding motif controlled the activation of endogenous Racl to induce membrane ruffling during HBV infection (21).

In addition, HBx expressing HepG 2 cells have been observed with long pseudopods (23). Furthermore, the morphological changes observed in hepatoma cells expressing HBx are associated with the microfilament bundles aligning along the pseudopods, and microtubules orienting towards the pseudopods (24). The Rho GTPase family proteins, including
Rac and Cdc42, are involved in these HBx-induced cellular polarized morphologies with pseudopods (24). Taken together, these findings indicate that the Rho GTPase family proteins serve an important role in $\mathrm{HBx}$-induced morphogenesis with the appearance of membrane ruffling and pseudopods during HBV infection (Fig. 1). However, the precise mechanisms underlying the HBx-regulated activity of Rho GTPase family proteins remain to be elucidated and require further study.

Epithelial-mesenchymal transition (EMT) has been increasingly recognized as a significant event in the development of HCC (25). EMT is the process by which epithelial cells undergo morphogenetic changes to acquire features of a fibroblastoid or mesenchymal cellular phenotype during the process of cancer progression (25-27). During EMT, the expression of E-cadherin, an epithelial cell-cell adhesion molecule, is downregulated, whilst the expression of the mesenchymal cell adhesion molecule $\mathrm{N}$-cadherin is upregulated in a process known as the cadherin switch (25). Sequentially, the cytoskeletal actin component $\beta$-catenin is released from the cellular membrane to enter the cytoplasmic pool (25-27). Following this, cytosolic $\beta$-catenin may be degraded or translocated into the nucleus (25). Additionally, enhanced expression of the cytoskeletal protein vimentin has been observed during the process of EMT in tumor cells $(25,26)$. A previous study has demonstrated that the hepatocytes observed in well-differentiated HCC samples are associated with E-cadherin at the plasma membrane, whereas the loss of E-cadherin expression is exhibited in poorly differentiated HCC (26). In addition, the nuclear translocation of $\beta$-catenin is accompanied by reduced E-cadherin expression levels. Furthermore, the expression levels of vimentin are also increased in poorly differentiated HCC tissue samples (26,27). Whilst a high load of HBV replication inhibits the EMT of HCC cells (28), HBx is considered to contribute to the promotion of EMT. HBx may be able to induce an EMT phenotype in hepatoma cells, with decreased E-cadherin expression levels as well as an upregulation of vimentin and N-cadherin (29). In addition, $\beta$-catenin is primarily observed in the cytoplasm and nuclei of HBx-transfected cells (30). Furthermore, HBx has been demonstrated to induce EMT in human hepatoma cells by activating proto-oncogene tyrosine-protein kinase (c-Src) (29), or by modulating the signal transducer and activator of transcription (STAT) 3 signaling pathway in order to activate Twist (31). HBx stimulation of the STAT5b signaling pathway, or the epigenetic silencing of secreted frizzled-related proteins 1 and 5 using hypermethylation, was also correlated with EMT in HBV-associated HCC tissue samples $(32,33)$. In addition, the stabilization of Snail protein via the activation of the phosphatidylinositol 3-kinase/protein

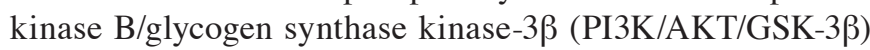
signaling pathway is involved in HBx induced EMT (34).

The underlying mechanisms involved in the cytoskeletal protein expression alterations associated with EMT, and those that are deregulated by $\mathrm{HBx}$, have previously been investigated in various studies (35-42). HBx has been demonstrated to downregulate E-cadherin via a range of mechanisms, including methylation of the E-cadherin promoter through DNA methyltransferase 1 activation (35), histone deacetylation of E-cadherin via recruitment of the $\mathrm{mSin} 3 \mathrm{~A} /$ histone 


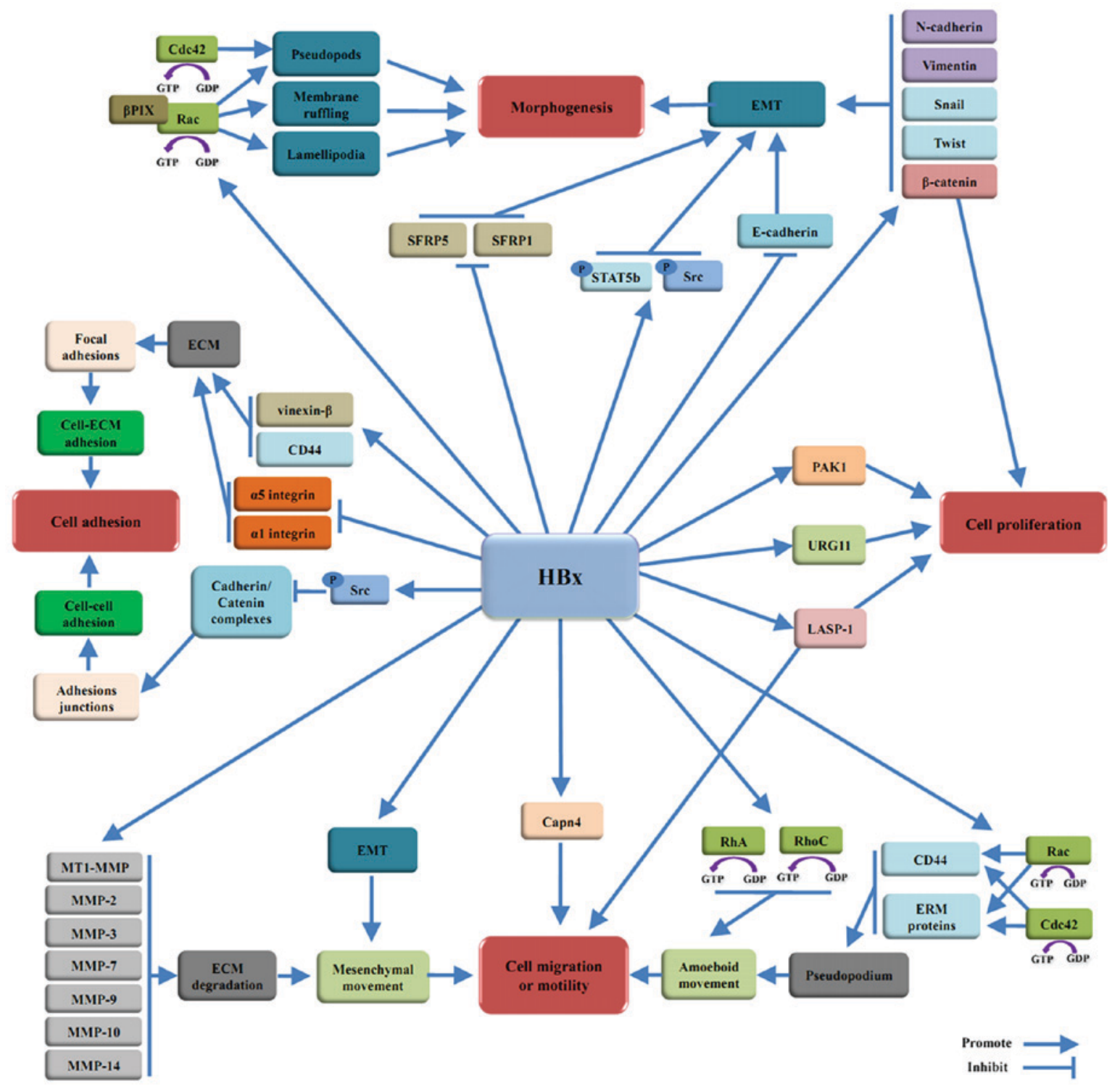

Figure 1. HBx has a role in the morphogenesis, adhesion, migration and proliferation of hepatoma cells. HBx is a multifunctional factor that primarily induces the change of cell morphogenesis with the appearance of lamellipodia, membrane ruffling, pseudopods and EMT. The abnormality of HBx-mediated cell adhesion relies on the disruption of cell-cell adhesion and cell-extracellular matrix adhesion. The enhanced movement or migration of HCC cells mediated by HBx is primarily dependent on mesenchymal and amoeboid migration. In addition, cytoskeletal proteins, including LASP-1 and Capn4, are involved in HBx-associated HCC cell migration. Increased HBx-promoted HCC proliferation is associated with various cytoskeletal proteins, including LASP-1, PAK1, $\beta$-catenin and URG11. HBx, hepatitis B virus X-protein; HCC, hepatocellular carcinoma; Cdc42, cell division control protein 42; $\beta$ PIX, Rho guanine nucleotide exchange factor 7; Rac, Ras-related C3 botulinum toxin; EMT, epithelial-mesenchymal transition; Src, proto-oncogene tyrosine-protein kinase Src; STAT5b, signal transducer and activator of transcription 5B; SFRP, secreted frizzled-related protein 1; PAK1, serine/threonine p21-activated kinases; URG11, upregulated gene 11; LASP-1, LIM and SH3 domain protein 1; Capn4, calpain small subunit 1; MT1, membrane type 1; MMP, matrix metalloproteinase; RhA, ATP-dependent RNA helicase A; RhoC, Ras homolog gene family, member C.

deacetylase 1 complex to the E-cadherin promoter (36) or the downregulation of microRNA-373 in HCC cells (36). In addition, HBx was determined to upregulate the expression of $\beta$-catenin in various ways. Firstly, HBx has been reported to stabilize cytoplasmic $\beta$-catenin through glycogen synthase kinase $3 \beta$ suppression, which occurs via the activation of $\mathrm{Src}$ kinase (37). Secondly, it has been demonstrated that HBx upregulates $\beta$-catenin by attenuating its interaction with silent mating type information regulation 2 homolog 1 (38). Thirdly, HBx activates the $\beta$-catenin promoter and accelerates $\beta$-catenin expression through increasing upregulated gene 11 (URG11) expression levels $(39,40)$. Hsieh et al (41) demonstrated that HBx binds adenomatous polyposis coli, displacing $\beta$-catenin from its degradation complex. Additionally, it has been revealed that HBx inhibits the expression of HBX-related long noncoding RNA downregulated expression by HBx to upregulate vimentin expression (42). EMT is a complex process mediated by various genes (26). The majority of published studies have focused primarily on known EMT-associated genes mediated by HBx in HBV-associated HCC (Fig. 1); however, a precise network of EMT-associated genes regulated by HBx has yet to be generated. Therefore, further studies are required to clarify the impact of HBx on the expression of EMT-associated genes at the genome-wide level, in order to find key targets for the clinical treatment of HBV-associated HCC. 


\section{The cellular cytoskeletal proteins associated with cell adhesion}

Tight cell-cell and cell-extracellular matrix (ECM) connections are required for the formation of a polarized and stable epithelial cell (43). Cell-cell adhesion is attained as a result of various structures, including adherens junctions, tight junctions and desmosomes (43). A family of transmembrane glycoproteins known as cadherins mediates the adherens junctions (44). A complex of numerous proteins mediates the anchorage of cadherins to adherens junctions, including actinin, vinculin and catenins $(43,44)$. The role of HBx in the disruption of cell-cell adhesion has not yet been elucidated; however, Lara-Pezzi et al (44) demonstrated that HBx is able to dissociate cadherin/catenin complexes to induce the disruption of adherens junctions in an Src kinase-dependent manner (Fig. 1).

Cell-ECM adhesion is predominantly regulated by various adhesion receptors, including integrins, to form focal adhesions (45). Integrins are heterodimeric transmembrane adhesion receptors composed of $18 \alpha$-subunits and 8 $\beta$-subunits, and may recognize specific peptide motifs located in ECM components, including fibronectin, vitronectin, laminin, hyaluronan and collagen $(46,47)$. HBV replication reduces the formation of focal adhesions and the actin concentration at the cell periphery, resulting in a significant reduction of adhesion to laminin in cells infected with HBV (48). Additionally, a decrease in cell adhesion to collagen is observed in HBV-replicating cells, as well as cells transfected with HBx (49). Furthermore, HBx has been demonstrated to interact with the focal adhesion protein vinexin- $\beta$ via binding to the SH3 domain, and the decrease of cell adhesion dynamics to the ECM may be associated with an interaction between vinexin- $\beta$ and $\mathrm{HBx}$ (49). HBx-expressing cells also demonstrate decreased adhesion to fibronectin, which correlates with decreased expression levels of the HBx-induced $\alpha 5$ integrin subunit (50). Additionally, a decrease in the expression levels of the collagen/laminin receptor $\alpha 1$ integrin subunit in cells expressing HBx has been observed (50). However, HBx-expressing cells exhibit a higher capacity to adhere to hyaluronan in a CD44-dependent manner (24). Together, these changes in the cell-ECM association mediated by $\mathrm{HBx}$ contribute to the cell adhesion abnormalities observed in HCC (Fig. 1) (49,50). Further understanding of the molecular mechanisms underlying HBx-mediated disruption of the cell-ECM connection may facilitate the discovery of novel therapeutic strategies.

\section{The cellular cytoskeletal proteins associated with cel- lular migration}

Tumor cells at various differentiation levels demonstrate migration and motility in numerous ways (51-53). In well-differentiated tumor cells, stable cell-cell contact forms a cohesive multicellular unit and cells undergo collective migration, forming what is known as a sheet-like structure (52). When tumor cells undergo EMT and acquire a mesenchymal phenotype, cell-cell contacts disintegrate and cells are able to migrate individually $(51,52)$. Depending on the strength of the adhesion between the tumor cells and ECM, individual cells may undergo amoeboid or mesenchymal migration (51). In the case of mesenchymal movement, cells form focal adhesions with ECM components and the movement of tumor cells is primarily dependent on the degradation of ECM (52). During amoeboid movement, focal adhesions between cells and components of the ECM are not formed, and the cell may migrate through the space between the ECM components $(51,52)$. In addition, tumor cells are able to switch from mesenchymal movement to amoeboid movement in response to microenvironmental stimulation, in a process known as mesenchymal-amoeboid transition (53).

As aforementioned, $\mathrm{HBx}$ is responsible for inducing the process of EMT in HCC cells, promoting their invasion and migration (29,31,32). Furthermore, upon the progression of HCC, numerous types of ECM-degrading enzymes, including matrix metalloproteinases (MMPs), are upregulated and activated by $\mathrm{HBx}$ to induce ECM degradation (54-57). Firstly, these findings indicate that $\mathrm{HCC}$ cells expressing $\mathrm{HBx}$ are associated with an increase in membrane-type 1 (MT1)-MMP and MMP-2 expression levels $(54,55)$. Secondly, the HBx-induced migratory phenotype in hepatoma cells is associated with the activation of MMP-3 (56). Thirdly, HBx induces MMP-9 expression via the activation of extracellular signal-related kinases (ERKs) and the PI3K/AKT signaling pathways (57).

The upregulation of MMP-9 and MMP-14, mediated by $\mathrm{HBx}$, is associated with the increased activation of the nuclear factor $\kappa \mathrm{B}(\mathrm{NF} \kappa \beta)$ signaling pathway in HepG2 cells (58). In addition, HBx has been demonstrated to stabilize amplified in breast 1 protein to enhance MMP-9 promoter activity and increase MMP-9 expression levels (59). Furthermore, Xia et al (60) suggested that HBx upregulates forkhead box protein M1 (FoxM1) expression through the ERK/cAMP response element-binding protein signaling pathway in order to facilitate MMP-2, -7 and -9 expression. In addition, C-terminal truncated HBx is implicated in HBV-associated HCC and correlated with hepatocarcinogenesis (7). Sze et al (61) demonstrated that $\mathrm{C}$-terminal truncated $\mathrm{HBx}$ was able to activate MMP-10 via c-Jun transcriptional activity. As $\mathrm{HBx}$-associated MMPs serve key roles in inducing ECM degradation to improve cell migration (Fig. 1), it is possible to exploit the inhibition of these MMPs by using blocking antibodies or small molecule inhibitors as potentially effective therapeutic strategies to control abnormal cell migration mediated by HBV infection.

Amoeboid movement depends on cellular polarization through the formation of anterior pseudopodia in order to move the cell forward $(51,52)$. HBx-induced cell migration is associated with the formation of a pseudopodium and the redistribution of activated $\beta 1$ subunits, CD44 and ezrin-radixin-moesin (ERM) proteins, particularly moesin, to the tips of the pseudopodium (24). This study also demonstrated that Rho GTPase family proteins, including Rac and Cdc42, were involved in cell polarization with pseudopodia, and that CD44 and ERM proteins redistribute in HBX expressing cells (24). Ezrin, a member of the ERM protein family, has also been observed to be overexpressed in patients with $\mathrm{HBV}$-associated HCC, and contribute to a higher migration and invasion capacity in hepatoma cells (62). Among the molecules in the Rho GTPase family, Racl could be activated by the replication of $\mathrm{HBV}$, resulting in the abnormal 
migration of HBV-replicating cells (63). Additionally, RhoA has been demonstrated to be activated by HBx to promote cell migration (64). RhoC, a member of the Rho subfamily, is also involved in the migration of HBV-associated HCC cells, and HBx-mediated overexpression of FoxM1 or Ets-1 is responsible for upregulation of $\mathrm{RhoC}(60,65,66)$. Due to the roles of Rho GTPase family proteins in $\mathrm{HBx}$-induced cell migration, these proteins may also be viable targets for HBV-associated HCC therapy.

In addition to the aforementioned proteins, increased expression levels of certain cell migration-associated proteins, including Ras GTPase-activating-like protein, annexin A2 and protein disulfide-isomerase $\mathrm{A} 3$ were identified by a proteomics study of HBV-expressing HepG2 cells, with the majority of these proteins being cytoskeletal (67). LIM and SH3 domain protein 1 (LASP-1) is a cytoskeletal protein that localizes to numerous dynamic actin assembly sites, including focal adhesions, pseudopodia, lamellipodia and membrane ruffles, and by reacting with various other cytoskeletal proteins, it is also involved in HBx-induced cell migration (23). HBx-mediated upregulation of LASP-1 expression occurs partially through the PI3K signaling pathway (23). In addition, previous studies indicated that HBx increases the expression levels of calpain small subunit 1 (Capn4), a protein belonging to the calpain family of calcium-dependent proteases, which is involved in focal adhesion dynamics $(68,69)$; it was revealed that Capn 4 is involved in HepG2 cell migration, and the increased activation of $\mathrm{NF} \kappa \beta / \mathrm{p} 65$ was demonstrated to be crucial in the regulation of Capn4 expression $(68,69)$.

\section{The cellular cytoskeletal proteins associated with cellu- lar proliferation}

Despite advancements in methods of detecting the dysregulation of cytoskeleton-associated proteins mediated by HBx, the role of these proteins during $\mathrm{HCC}$ cell proliferation is still poorly understood (9-15). Xu et al (70) suggested that HBx may upregulate serine/threonine $\mathrm{p} 21$-activated kinase 1 , which is involved in the regulation of cytoskeletal dynamics and the protection of cells from anoikis, in order to promote the growth of tumors in mice. $\mathrm{HBx}$ also promotes cell proliferation via the upregulation of LASP-1, and inhibits the expression of LASP-1 to decrease the accumulation of $\mathrm{HBx}$ bearing cells at the $\mathrm{G} 2 / \mathrm{M}$ phase of the cell cycle (23). Furthermore, $\beta$-catenin serves an essential role in the hepatoma cell proliferation induced by HBx (71). URG11 is a protein that is associated with $\beta$-catenin expression in HBx-associated HCC cells (39), which is able to stimulate cell growth and cell cycle progression in vitro. In addition, URG11 stimulates anchorage-independent cell growth in soft agar and HBx-mediated tumor formation in severe combined immunodeficiency mice (72). Considering the vital role of cytoskeletal proteins in cell proliferation (Fig. 1), further studies are required in order to elucidate how HBx-associated cytoskeletal proteins regulate cell proliferation during HBV infection.

\section{Conclusion}

In conclusion, $\mathrm{HBV}$-associated hepatocarcinogenesis is regarded as a multifactorial process; however, the exact underlying mechanisms remain to be established. Previous studies have suggested that $\mathrm{HBx}$-mediated cytoskeletal proteins are involved in hepatocarcinogenesis and participate in cell morphogenesis, adhesion, migration and proliferation via various mechanisms (Fig. 1). Although the roles of a number of cytoskeletal genes and proteins mediated by HBx have not been determined, the reviewed studies provide insights into the function of these factors in the development of HBV-associated HCC. Chemotherapeutic agents that are able to induce stabilization of cytoskeleton-associated proteins to restrict movement, as well as limit intracellular processes, have been studied as potential therapeutic agents for certain tumors $(46,73)$. Therefore, it is possible that clarifying the underlying regulatory mechanisms of cytoskeleton-associated proteins during HBV infection may facilitate the identification of novel potential therapeutic targets for HBV-associated HCC.

\section{Acknowledgements}

The present study was supported by the research funding of Jiangsu Key Laboratory of Brain Disease Bioinformation (grant no. Jsb11401), the Scientific Research Foundation for the Talents of Xuzhou Medical University (grant no. D2016011), the Qing Lan Project of Jiangsu province and The Priority Academic Program Development of Jiangsu Higher Education Institutions (PAPD).

\section{References}

1. Rivière L, Ducroux A and Buendia MA: The oncogenic role of hepatitis B virus. Recent Results Cancer Res 193: 59-74, 2014.

2. Feitelson MA, Bonamassa B and Arzumanyan A: The roles of hepatitis B virus-encoded $X$ protein in virus replication and the pathogenesis of chronic liver disease. Expert Opin Ther Targets 18: 293-306, 2014.

3. Xie N, Chen X, Zhang T, Liu B and Huang C: Using proteomics to identify the $\mathrm{HBx}$ interactome in hepatitis B virus: How can this inform the clinic? Expert Rev Proteomics 11: 59-74, 2014.

4. Zhang X, Zhang $\mathrm{H}$ and Ye L: Effects of hepatitis B virus X protein on the development of liver cancer. J Lab Clin Med 147: 58-66, 2006.

5. Ng SA and Lee C: Hepatitis B virus X gene and hepatocarcinogenesis. J Gastroenterol 46: 974-990, 2011.

6. Tian Y, Yang W, Song J, Wu Y and Ni B: Hepatitis B virus X protein-induced aberrant epigenetic modifications contributing to human hepatocellular carcinoma pathogenesis. Mol Cell Biol 33: 2810-2816, 2013

7. Ali A, Abdel-Hafiz H, Suhail M, Al-Mars A, Zakaria MK, Fatima K, Ahmad S, Azhar E, Chaudhary A and Qadri I: Hepatitis B virus, HBx mutants and their role in hepatocellular carcinoma. World J Gastroenterol 20: 10238-10248, 2014.

8. Nakanishi F, Ohkawa K, Ishida H, Hosui A, Sato A, Hiramatsu N, Ueda K, Takehara T, Kasahara A, Sasaki Y, et al: Alteration in gene expression profile by full-length hepatitis B virus genome. Intervirology 48: 77-83, 2005.

9. Wu CG, Salvay DM, Forgues M, Valerie K, Farnsworth J, Markin RS and Wang XW: Distinctive gene expression profiles associated with Hepatitis B virus x protein. Oncogene 20: 3674-3682, 2001.

10. Sun Q, Wang Y, Zhang Y, Liu F, Cheng X, Hou N, Zhao X and Yang $X$ : Expression profiling reveals dysregulation of cellular cytoskeletal genes in $\mathrm{HBx}$-induced hepatocarcinogenesis. Cancer Biol Ther 6: 668-674, 2007.

11. Zhang WY, Xu FQ, Shan CL, Xiang R, Ye LH and Zhang XD: Gene expression profiles of human liver cells mediated by hepatitis B virus X protein. Acta Pharmacol Sin 30: 424-434, 2009.

12. Ng RK, Lau CY, Lee SM, Tsui SK, Fung KP and Waye MM: cDNA microarray analysis of early gene expression profiles associated with hepatitis B virus $X$ protein-mediated hepatocarcinogenesis. Biochem Biophys Res Commun 322: 827-835, 2004. 
13. Ye L, Dong N, Wang Q, Xu Z, Cai N, Wang H and Zhang X: Progressive changes in hepatoma cells stably transfected with hepatitis B virus X gene. Intervirology 51: 50-58, 2008.

14. Sung WK, Lu Y, Lee CW, Zhang D, Ronaghi M and Lee CG: Deregulated direct targets of the hepatitis B virus (HBV) protein $\mathrm{HBx}$, identified through chromatin immunoprecipitation and expression microarray profiling. J Biol Chem 284: 21941-21954, 2009.

15. Feng H, Li X, Niu D and Chen WN: Protein profile in HBx transfected cells: A comparative iTRAQ-coupled 2D LC-MS/MS analysis. J Proteomics 73: 1421-1432, 2010.

16. Wang J, Jiang D, Zhang H, Lv S, Rao H, Fei R and Wei L: Proteome responses to stable hepatitis B virus transfection and following interferon alpha treatment in human liver cell line HepG2. Proteomics 9: 1672-1682, 2009.

17. Stringham EG, Marcus-Gueret N, Ramsay L and Schmidt KL: Live cell imaging of the cytoskeleton. Methods Enzymol 505: 203-217, 2012

18. Hilfer SR and Searls RL: Cytoskeletal dynamics in animal morphogenesis. Dev Biol (N Y 1985) 2: 3-29, 1986.

19. Meuleman P, Libbrecht L, Wieland S, De Vos R, Habib N, Kramvis A, Roskams T and Leroux-Roels G: Immune suppression uncovers endogenous cytopathic effects of the hepatitis B virus. J Virol 80: 2797-2807, 2006.

20. Hirschman SZ, Price P, Garfinkel E, Christman J and Acs G: Expression of cloned hepatitis B virus DNA in human cell cultures. Proc Natl Acad Sci USA 77: 5507-5511, 1980.

21. Tan TL, Fang N, Neo TL, Singh P, Zhang J, Zhou R, Koh CG, Chan V, Lim SG and Chen WN: Racl GTPase is activated by hepatitis B virus replication-involvement of HBX. Biochim Biophys Acta 1783: 360-374, 2008.

22. Vega FM and Ridley AJ: Rho GTPases in cancer cell biology. FEBS Lett 582: 2093-2101, 2008.

23. Tang R, Kong F, Hu L, You H, Zhang P, Du W and Zheng K: Role of hepatitis B virus X protein in regulating LIM and SH3 protein 1 (LASP-1) expression to mediate proliferation and migration of hepatoma cells. Virol J 9: 163, 2012.

24. Lara-Pezzi E, Serrador JM, Montoya MC, Zamora D, Yáñez-Mó M, Carretero M, Furthmayr H, Sánchez-Madrid F and López-Cabrera M: The hepatitis B virus X protein $(\mathrm{HBx})$ induces a migratory phenotype in a CD44-dependent manner: Possible role of HBx in invasion and metastasis. Hepatology 33 1270-1281, 2001

25. Pogribny IP and Rusyn I: Role of epigenetic aberrations in the development and progression of human hepatocellular carcinoma. Cancer Lett 342: 223-230, 2014.

26. van Zijl F, Zulehner G, Petz M, Schneller D, Kornauth C Hau M, Machat G, Grubinger M, Huber H and Mikulits W: Epithelial-mesenchymal transition in hepatocellular carcinoma. Future Oncol 5: 1169-1179, 2009.

27. Ogunwobi $\mathrm{OO}$ and Liu C: Therapeutic and prognostic importance of epithelial-mesenchymal transition in liver cancers Insights from experimental models. Crit Rev Oncol Hematol 83: 319-328, 2012

28. Wang T, Jin Y, Zhao R, Wu Y, Zhang Y, Wu D, Kong D, Jin X and Zhang F: High load hepatitis B virus replication inhibits hepatocellular carcinoma cell metastasis through regulation of epithelial-mesenchymal transition. Int J Infect Dis 20: 37-41, 2014.

29. Yang SZ, Zhang LD, Zhang Y, Xiong Y, Zhang YJ, Li HL, Li XW and Dong JH: HBx protein induces EMT through c-Src activation in SMMC-7721 hepatoma cell line. Biochem Biophys Res Commun 382: 555-560, 2009.

30. Liu J, Lian Z, Han S, Waye MM, Wang H, Wu MC, Wu K, Ding J, Arbuthnot P, Kew M, et al: Downregulation of E-cadherin by hepatitis B virus $\mathrm{X}$ antigen in hepatocellullar carcinoma. Oncogene 25: 1008-1017, 2006

31. Teng J, Wang X, Xu Z and Tang N: HBx-dependent activation of Twist mediates STAT3 control of epithelium-mesenchymal transition of liver cells. J Cell Biochem 114: 1097-1104, 2013

32. Lee TK, Man K, Poon RT, Lo CM, Yuen AP, Ng IO, Ng KT, Leonard W and Fan ST: Signal transducers and activators of transcription $5 \mathrm{~b}$ activation enhances hepatocellular carcinoma aggressiveness through induction of epithelial-mesenchyma transition. Cancer Res 66: 9948-9956, 2006.

33. Xie Q, Chen L, Shan X, Shan X, Tang J, Zhou F, Chen Q, Quan H, Nie D, Zhang W, et al: Epigenetic silencing of SFRP1 and SFRP5 by hepatitis B virus X protein enhances hepatoma cell tumorigenicity through Wnt signaling pathway. Int J Cancer 135: 635-646, 2014 .
34. Liu H, Xu L, He H, Zhu Y, Liu J, Wang S, Chen L, Wu Q, Xu J and $\mathrm{Gu}$ J: Hepatitis $\mathrm{B}$ virus $\mathrm{X}$ protein promotes hepatoma cell invasion and metastasis by stabilizing Snail protein. Cancer Sci 103: 2072-2081, 2012

35. Lee JO, Kwun HJ, Jung JK, Choi KH, Min DS and Jang KL: Hepatitis B virus X protein represses E-cadherin expression via activation of DNA methyltransferase 1. Oncogene 24: 6617-6625, 2005.

36. Arzumanyan A, Friedman T, Kotei E, Ng IO, Lian Z and Feitelson MA: Epigenetic repression of E-cadherin expression by hepatitis B virus $x$ antigen in liver cancer. Oncogene 31: 563-572, 2012.

37. Cha MY, Kim CM, Park YM and Ryu WS: Hepatitis B virus X protein is essential for the activation of Wnt/beta-catenin signaling in hepatoma cells. Hepatology 39: 1683-1693, 2004

38. Srisuttee R, Koh SS, Kim SJ, Malilas W, Boonying W, Cho IR, Jhun BH, Ito M, Horio Y, Seto E, et al: Hepatitis B virus X (HBX) protein upregulates $\beta$-catenin in a human hepatic cell line by sequestering SIRT1 deacetylase. Oncol Rep 28: 276-282, 2012.

39. Lian Z, Liu J, Li L, Li X, Clayton M, Wu MC, Wang HY, Arbuthnot P, Kew M, Fan D and Feitelson MA: Enhanced cell survival of Hep3B cells by the hepatitis B $\mathrm{x}$ antigen effector, URG11, is associated with upregulation of beta-catenin. Hepatology 43: 415-424, 2006.

40. Du R, Huang C, Bi Q, Zhai Y, Xia L, Liu J, Sun S and Fan D URG11 mediates hypoxia-induced epithelial-to-mesenchymal transition by modulation of E-cadherin and beta-catenin. Biochem Biophys Res Commun 391: 135-141, 2010.

41. Hsieh A, Kim HS, Lim SO, Yu DY and Jung G: Hepatitis B viral $\mathrm{X}$ protein interacts with tumor suppressor adenomatous polyposis coli to activate Wnt/ $\beta$-catenin signaling. Cancer Lett 300: $162-172,2011$.

42. Huang JF, Guo YJ, Zhao CX, Yuan SX, Wang Y, Tang GN, Zhou WP and Sun SH: Hepatitis B virus X protein (HBx)-related long noncoding RNA (lncRNA) down-regulated expression by HBx (Dreh) inhibits hepatocellular carcinoma metastasis by targeting the intermediate filament protein vimentin. Hepatology 57: 1882-1892, 2013

43. Andl CD: The misregulation of cell adhesion components during tumorigenesis: Overview and commentary. J Oncol 2010: 174715, 2010.

44. Lara-Pezzi E, Roche S, Andrisani OM, Sánchez-Madrid F and López-Cabrera M: The hepatitis B virus HBx protein induces adherens junction disruption in a src-dependent manner. Oncogene 20: 3323-3331, 2001

45. Ciobanasu C, Faivre B and Le Clainche C: Integrating actin dynamics, mechanotransduction and integrin activation: The multiple functions of actin binding proteins in focal adhesions. Eur J Cell Biol 92: 339-348, 2013.

46. Kapp TG, Rechenmacher F, Sobahi TR and Kessler H: Integrin modulators: A patent review. Expert Opin Ther Pat 23: 1273-1295, 2013

47. Frantz C, Stewart KM and Weaver VM: The extracellular matrix at a glance. J Cell Sci 123: 4195-4200, 2010.

48. Li X, Feng H, Chen WN and Chan V: Hepatitis B virus induced coupling of deadhesion and migration of HepG2 cells on thermo-responsive polymer. Biomaterials 31: 1894-1903, 2010.

49. Tan TL, Feng Z, Lu YW, Chan V and Chen WN: Adhesion contact kinetics of HepG2 cells during Hepatitis B virus replication: Involvement of SH3-binding motif in HBX. Biochim Biophys Acta 1762: 755-766, 2006.

50. Lara-Pezzi E, Majano PL, Yáñez-Mó M, Gómez-Gonzalo M, Carretero M, Moreno-Otero R, Sánchez-Madrid F and López-Cabrera M: Effect of the hepatitis B virus HBx protein on integrin-mediated adhesion to and migration on extracellular matrix. J Hepatol 34: 409-415, 2001

51. Dvorakova M, Nenutil R and Bouchal P: Transgelins, cytoskeletal proteins implicated in different aspects of cancer development. Expert Rev Proteomics 11: 149-165, 2014.

52. Friedl P and Wolf K: Plasticity of cell migration: A multiscale tuning model. J Cell Biol 188: 11-19, 2010.

53. Wolf K, Mazo I, Leung H, Engelke K, von Andrian UH, Deryugina EI, Strongin AY, Bröcker EB and Friedl P: Compensation mechanism in tumor cell migration: Mesenchymal-amoeboid transition after blocking of pericellular proteolysis. J Cell Biol 160: 267-277, 2003

54. Ou DP, Tao YM, Tang FQ and Yang LY: The hepatitis B virus $\mathrm{X}$ protein promotes hepatocellular carcinoma metastasis by upregulation of matrix metalloproteinases. Int J Cancer 120: $1208-1214,2007$ 
55. Lara-Pezzi E, Gómez-Gaviro MV, Gálvez BG, Mira E, Iñiguez MA, Fresno M, Martínez-A C, Arroyo AG and López-Cabrera M: The hepatitis $\mathrm{B}$ virus $\mathrm{X}$ protein promotes tumor cell invasion by inducing membrane-type matrix metalloproteinase-1 and cyclooxygenase-2 expression. J Clin Invest 110: $1831-1838,2002$

56. Yu FL, Liu HJ, Lee JW, Liao MH and Shih WL: Hepatitis B virus $\mathrm{X}$ protein promotes cell migration by inducing matrix metalloproteinase-3. J Hepatol 42: 520-527, 2005.

57. Chung TW, Lee YC and Kim CH: Hepatitis B viral HBx induces matrix metalloproteinase-9 gene expression through activation of ERK and PI-3K/AKT pathways: Involvement of invasive potential. FASEB J 18: 1123-1125, 2004.

58. Liu LP, Liang HF, Chen XP, Zhang WG, Yang SL, Xu T and Ren L: The role of NF-kappaB in Hepatitis b virus X protein-mediated upregulation of VEGF and MMPs. Cancer Invest 28: 443-451, 2010.

59. Liu Y, Tong Z, Li T, Chen Q, Zhuo L, Li W, Wu RC and Yu C: Hepatitis B virus $\mathrm{X}$ protein stabilizes amplified in breast cancer 1 protein and cooperates with it to promote human hepatocellular carcinoma cell invasiveness. Hepatology 56: 1015-1024, 2012.

60. Xia L, Huang W, Tian D, Zhu H, Zhang Y, Hu H, Fan D, Nie Y and Wu K: Upregulated FoxM1 expression induced by hepatitis B virus $\mathrm{X}$ protein promotes tumor metastasis and indicates poor prognosis in hepatitis B virus-related hepatocellular carcinoma. J Hepatol 57: 600-612, 2012.

61. Sze KM, Chu GK, Lee JM and Ng IO: C-terminal truncated hepatitis $\mathrm{B}$ virus $\mathrm{x}$ protein is associated with metastasis and enhances invasiveness by C-Jun/matrix metalloproteinase protein 10 activation in hepatocellular carcinoma. Hepatology 57: 131-139, 2013.

62. Yeh CN, Pang ST, Chen TW, Wu RC, Weng WH and Chen MF: Expression of ezrin is associated with invasion and dedifferentiation of hepatitis B related hepatocellular carcinoma. BMC Cancer 9: 233, 2009.

63. Feng H, Zhang J, Li X and Chen WN: HBX-mediated migration of HBV-replicating HepG2 cells: Insights on development of hepatocellular carcinoma. J Biomed Biotechnol 2009: 930268, 2009.
64. Feng H, Li X, Niu D and Ning Chen W: HBx expression activates RhoA GTPase: Impact on cell migration. Cell Biol Int 35: 159-164, 2011.

65. Tian Y, Liu Y, Qu J, Li K, Qin D, Huang A and Tang H: HBV regulated RhoC expression in HepG2.2.15 cells by enhancing its promoter activity. J Basic Microbiol 53: 461-468, 2013.

66. Qin D, Li K, Qu J, Wang S, Zou C, Sheng Y, Huang A and Tang $\mathrm{H}$ : $\mathrm{HBx}$ and $\mathrm{HBs}$ regulate RhoC expression by upregulating transcription factor Ets-1. Arch Virol 158: 1773-1781, 2013.

67. Feng H, Li X, Chan V and Chen WN: Proteomics based identification of cell migration related proteins in HBV expressing HepG2 cells. PLoS One 9: e95621, 2014.

68. Zhang F, Wang Q, Ye L, Feng Y and Zhang X: Hepatitis B virus $\mathrm{X}$ protein upregulates expression of calpain small subunit 1 via nuclear factor-kappaB/p65 in hepatoma cells. J Med Virol 82: 920-928, 2010

69. Zhang X, You X, Wang Q, Zhang T, Du Y, Lv N, Zhang Z, Zhang S, Shan C, Ye L and Zhang X: Hepatitis B virus X protein drives multiple cross-talk cascade loops involving NF- $\kappa \mathrm{B}$, 5-LOX, OPN and Capn4 to promote cell migration. PLoS One 7: e31458, 2012.

70. Xu J, Liu H, Chen L, Wang S, Zhou L, Yun X, Sun L, Wen Y and $\mathrm{Gu}$ J: Hepatitis B virus $X$ protein confers resistance of hepatoma cells to anoikis by up-regulating and activating p21-activated kinase 1. Gastroenterology 143: 199-212.e194, 2012.

71. Sun Q, Wang R, Luo J, Wang P, Xiong S, Liu M and Cheng B: Notch1 promotes hepatitis $\mathrm{B}$ virus $\mathrm{X}$ protein-induced hepatocarcinogenesis via Wnt/ $\beta$-catenin pathway. Int J Oncol 45: 1638-1648, 2014

72. Lian Z, Liu J, Li L, Li X, Tufan NL, Clayton M, Wu MC, Wang HY, Arbuthnot P, Kew M and Feitelson MA: Upregulated expression of a unique gene by hepatitis B x antigen promotes hepatocellular growth and tumorigenesis. Neoplasia 5: 229-244, 2003.

73. Martin SK, Kamelgarn M and Kyprianou N: Cytoskeleton targeting value in prostate cancer treatment. Am J Clin Exp Urol 2: 15-26, 2014. 